\title{
Approximation of common solutions for a fixed point problem of asymptotically nonexpansive mapping and a generalized equilibrium problem in Hilbert space
}

\author{
Richard Osward ${ }^{1}$, Santosh Kumar ${ }^{1 *}$ and Mengistu Goa Sangago ${ }^{2}$
}

\section{${ }^{*}$ Correspondence:}

drsengar2002@gmail.com 'Department of Mathematics, College of Natural and Applied Sciences, University of Dar es Salaam, Dar es Salaam, Tanzania Full list of author information is available at the end of the article

\begin{abstract}
In this paper, we introduce an iterative algorithm to approximate a common solution of a generalized equilibrium problem and a fixed point problem for an asymptotically nonexpansive mapping in a real Hilbert space. We prove that the sequences generated by the iterative algorithm converge strongly to a common solution of the generalized equilibrium problem and the fixed point problem for an asymptotically nonexpansive mapping. The results presented in this paper extend and generalize many previously known results in this research area. Some applications of main results are also provided.
\end{abstract}

Keywords: Fixed points, Generalized equilibrium problem, Asymptotically nonexpansive mapping, Iterative algorithm

Mathematics Subject Classification 2010: 47J05, 47J20, 47H09.

\section{Introduction}

Throughout the paper unless otherwise stated, let $H$ be a real Hilbert space with inner product $\langle.,$.$\rangle and induced norm \|$.$\| . Let C$ be a nonempty closed convex subsets of $H$. Let $\left\{x_{n}\right\}$ be a sequence in $H$, then $x_{n} \rightarrow x$ (respectively, $x_{n} \rightarrow x$ ) denotes strong (respectively, weak) convergence of the sequence $\left\{x_{n}\right\}$ to a point $x \in H$. We denote by $\mathbb{N}$ and $\mathbb{R}$ the sets of all positive integers and all real numbers, respectively. For every point $x \in H$, there exists a unique nearest point of $C$, denoted by $P_{C}$, such that

$$
\left\|x-P_{C} x\right\| \leq\|x-y\| \text { for all } y \in C .
$$

Such a $P_{C}$ is called the metric projection from $H$ onto $C$.

A mapping $T: C \rightarrow C$ is said to be asymptotically nonexpansive [1] if there exists a sequence $\left\{k_{n}\right\} \subset[1, \infty)$ with $\lim _{n \rightarrow \infty} k_{n}=1$ such that

$$
\left\|T^{n} x-T^{n} y\right\| \leq k_{n}\|x-y\|, \forall x, y \in C .
$$

$T$ is said to be a uniformly $k$-Lipschitzian for a positive constant $k$ if

$$
\left\|T^{n} x-T^{n} y\right\| \leq k\|x-y\|, \forall x, y \in C, \forall n \in \mathbb{N} .
$$

If $k_{n}=1, \forall n \in \mathbb{N}$, then $T$ is said to be a nonexpansive mapping. A point $x \in X$ is called a fixed point for $T$ if $T x=x$.

(c) The Author(s). 2019 Open Access This article is distributed under the terms of the Creative Commons Attribution 4.0 International License (http://creativecommons.org/licenses/by/4.0/), which permits unrestricted use, distribution, and reproduction in any medium, provided you give appropriate credit to the original author(s) and the source, provide a link to the Creative Commons license, and indicate if changes were made. 
The fixed point problem (in short, FPP) for the mapping $T: C \rightarrow C$ is to find $x \in C$ such that

$$
T x=x .
$$

The solution set of FPP (1) is denoted by $F(T)$, that is,

$$
F(T)=\{x \in C: T x=x\} .
$$

Let $F: C \times C \rightarrow \mathbb{R}$ be a bifunction and $A: C \rightarrow H$ be a nonlinear mapping. The generalized equilibrium problem is to find $z \in C$ such that

$$
F(z, y)+\langle A z, y-z\rangle \geq 0, \forall y \in C .
$$

The set of the solution of the problem (2) is denoted by $\operatorname{EP}(F, A)$, that is,

$$
E P(F, A)=\{z \in C: F(z, y)+\langle A z, y-z\rangle \geq 0, \forall y \in C\} .
$$

If $A \equiv 0$ in (2), then problem (2) reduces to the equilibrium problem of finding an element $z \in C$ such that,

$$
F(z, y) \geq 0, \forall y \in C .
$$

The set of solutions of problem (3) is denoted by $E P(F)$.

If $F \equiv 0$ in (2), then the generalized equilibrium problem (2) is reduced to finding a point $z \in C$ such that,

$$
\langle A z, y-z\rangle \geq 0, \forall y \in C,
$$

which is called the classical variational inequality problem. The set of solution of the problem (4) is denoted by $\operatorname{VI}(C, A)$.

If we define $F(x, y)=\langle A x, y-x\rangle$ for all $x, y \in C$, then $z \in E P(F)$ if and only if $\langle A z, y-z\rangle \geq 0$ for all $y \in C$ and hence $z \in V I(C, A)$.

The problem (2) is very general in the sense that it includes many special cases such as optimization problems, variational inequalities, minimax problems, and the Nash equilibrium problem in noncooperative games; see Blum and Oettli [2], Kazmi and Rizvi [3], Meche et al.[4], Moudafi and Théra [5], Zegeye et al. [6], and the references therein.

Throughout this paper, let us assume that a bifunction $F: C \times C \rightarrow \mathbb{R}$ satisfies the following conditions:

(A1) $\quad F(x, x)=0$ for all $x \in C$;

(A2) $\quad F$ is monotone, i.e., $F(x, y)+F(y, x) \leq 0$ for all $x, y \in C$;

(A3) for each $x, y, z \in C$;

$$
\lim _{t \downarrow 0} \sup F(t z+(1-t) x, y) \leq F(x, y) ;
$$

(A4) for each $x \in C, y \mapsto F(x, y)$ is convex and lower semi-continuous.

Definition 1 A mapping $A: C \rightarrow H$ is called $\alpha$-inverse strongly monotone if there exists a positive real number $\alpha$ such that,

$$
\langle A x-A y, x-y\rangle \geq \alpha\|A x-A y\|^{2}, \forall x, y \in C .
$$

Remark 1 Every $\alpha$-inverse strong monotone mapping is $\frac{1}{\alpha}$-Lipschitz mapping; however, the converse may not hold. 
Takahashi and Takahashi [7] obtained the following strong convergence theorem to find a common solution of generalized equilibrium problem and the fixed point problem of a nonexpansive mapping in a Hilbert space.

Theorem 1 [7] Let $C$ be a nonempty closed convex subset of a real Hilbert space H. Let $F: C \times C \rightarrow \mathbb{R}$ be a bifunction satisfying (A1), (A2), (A3), and (A4). Let $A: C \rightarrow H$ be an $\alpha$-inverse strongly monotone mapping, and let $T: C \rightarrow C$ be a nonepansive mapping such that $F(T) \cap E P(F, A) \neq \emptyset$. Let $u \in C$ and $x_{1} \in C$ and let $\left\{z_{n}\right\} \subset C$ and $\left\{x_{n}\right\} \subset C$ be sequence generated by

$$
\left\{\begin{array}{l}
F\left(z_{n}, y\right)+\left\langle A x_{n}, y-z_{n}\right\rangle+\frac{1}{\lambda_{n}}\left\langle y-z_{n}, z_{n}-x_{n}\right\rangle \geq 0, \forall y \in C, \\
x_{n+1}=\beta_{n} x_{n}+\left(1-\beta_{n}\right) T\left[\alpha_{n} u+\left(1-\alpha_{n}\right) z_{n}\right], \forall n \in \mathbb{N},
\end{array}\right.
$$

where $\left\{\alpha_{n}\right\} \subset[0,1],\left\{\beta_{n}\right\} \subset[0,1]$ and $\left\{\lambda_{n}\right\} \subset[0,2 \alpha]$ satisfy

1. $\lim _{n \rightarrow \infty} \alpha_{n}=0$ and $\sum_{n=1}^{\infty} \alpha_{n}=\infty$,

2. $\lim _{n \rightarrow \infty}\left(\lambda_{n}-\lambda_{n+1}\right)=0$, and

3. $0<c \leq \beta_{n} \leq d<1,0<a \leq \lambda_{n} \leq b<2 \alpha$.

Then, $\left\{x_{n}\right\}$ converges strongly to $z=P_{F(T) \cap E P(F, A)}(u)$.

In this paper, motivated by Takahashi and Takahashi [7], we construct an iterative algorithm for approximating a common solution of a generalized equilibrium problem and the fixed point problem for asymptotically nonexpansive mapping. It is also proved that the proposed algorithm converges strongly to a common solution.

\section{Preliminaries}

We now introduce preliminaries which will be used in this paper.

Recall that a mapping $f: C \rightarrow C$ is called a contraction mapping if there exists $\rho \in[0,1)$ such that

$$
\|f(x)-f(y)\| \leq \rho\|x-y\|, \forall x, y \in C .
$$

Lemma 1 [2] Let $C$ be a nonempty closed convex subset of a real Hilbert space H. Let $F: C \times C \rightarrow \mathbb{R}$ be a bifunction satisfying (A1), (A2), (A3), and (A4). Let $r>0$ and $x \in H$. Then, there exists $z \in C$ such that

$$
F(z, y)+\frac{1}{r}\langle y-z, z-x\rangle \geq 0, \forall y \in C .
$$

Lemma 2 [8] Let $C$ be a nonempty closed convex subset of $H$ and let $F: C \times C \rightarrow \mathbb{R}$ be a bi-function satisfying (A1), (A2), (A3), and (A4). Then, for any $r>0$ and $x \in H$, there exists $z \in C$ such that

$$
F(z, y)+\frac{1}{r}\langle y-z, z-x\rangle \geq 0, \quad \forall y \in C .
$$

Furthermore, if

$$
T_{r} x=\left\{z \in C: F(z, y)+\frac{1}{r}\langle y-z, z-x\rangle \geq 0, \forall y \in C\right\},
$$

then the following hold:

(1) $T_{r}$ is single valued, 
(2) $T_{r}$ is firmly non-expansive, i.e.,

$$
\left\|T_{r} x-T_{r} y\right\|^{2} \leq\left\langle T_{r} x-T_{r} y, x-y\right\rangle, \forall x, y \in H,
$$

(3) $F\left(T_{r}\right)=E P(F)$,

(4) $E P(F)$ is closed and convex.

Remark 2 Replacing $x$ with $x-r A x \in H$ in Lemma 1, there exists $z \in C$, such that

$$
F(z, y)+\langle A x, y-z\rangle+\frac{1}{r}\langle y-z, z-x\rangle \geq 0, \forall y \in C .
$$

Definition 2 [9] Let $C$ be a closed convex subset of a Hilbert space $H$. A mapping $T$ :

$C \rightarrow C$ is called asymptotically regular at $x$ if and only if,

$$
\lim _{n \rightarrow \infty}\left\|T^{n} x-T^{n+1} x\right\|=0 .
$$

Lemma 3 [10] Let $F: C \rightarrow C$ be a bifunction satisfying the conditions (A1) and (A2). Let $T_{r}$ and $T_{s}$ be defined as in Lemma 2 with $r, s>0$. For any $x, y \in H$, then

$$
\left\|T_{r} y-T_{s} x\right\| \leq\|y-x\|+\left|\frac{r-s}{r}\right|\left\|T_{r} y-y\right\| .
$$

Lemma 4 [11] Let $\left\{\delta_{n}\right\}$ be a sequence of non negative real numbers, satisfying

$$
\delta_{n+1} \leq\left(1-s_{n}\right) \delta_{n}+s_{n} \beta_{n}+\gamma_{n}, \forall n \geq 0,
$$

where $\left\{s_{n}\right\},\left\{\beta_{n}\right\}$ and $\left\{\gamma_{n}\right\}$ satisfies the conditions:

(i) $\left\{s_{n}\right\} \subset[0,1], \sum_{n=1}^{\infty} s_{n}=\infty$ or equivalently, $\prod_{n=1}^{\infty}\left(1-s_{n}\right)=0$,

(ii) $\lim \sup _{n \rightarrow \infty} \beta_{n} \leq 0$,

(iii) $\gamma_{n} \geq 0, \sum_{n=1}^{\infty} \gamma_{n} \leq \infty$

Then,

$$
\lim _{n \rightarrow \infty} \delta_{n}=0 .
$$

Lemma 5 [12] Let $T$ be an asymptotically nonexpansive mapping on a closed and convex subset $C$ of a real Hilbert space $H$. Then, $I-T$ is demiclosed at 0 . That is, for a sequence $\left\{x_{n}\right\}$ in $C$, if $x_{n} \rightarrow x$ and $x_{n}-T x_{n} \rightarrow 0$, then $x \in F(T)$.

Lemma 6 [13] Let $H$ be a real Hilbert space. Then, for any given $x, y \in H$, we have the following inequality:

$$
\|x+y\|^{2} \leq\|x\|^{2}+2\langle y, x+y\rangle .
$$

Lemma 7 [14] Let $\left\{t_{n}\right\}$ be a sequence of nonnegative real numbers such that

$$
t_{n+1} \leq\left(1-a_{n}\right) t_{n}+a_{n} \beta_{n}, \quad n \geq 0
$$

where $\left\{a_{n}\right\}$ is a sequence in $(0,1)$ and $\left\{\beta_{n}\right\}$ is a sequence in $\mathbb{R}$ such that

(C1) $\sum_{n=0}^{\infty} a_{n}=\infty$ or equivalently $\prod_{n=0}^{\infty}\left(1-a_{n}\right)=0$,

(C2) $\limsup _{n \rightarrow \infty} \beta_{n} \leq 0$. 
Then

$$
\lim _{n \rightarrow \infty} t_{n}=0
$$

\section{Main results}

Let $F: C \times C \rightarrow \mathbb{R}$ be a bifunction satisfying (A1), (A2), (A3), and (A4). Let $A: C \rightarrow H$ be $\alpha$-inverse strongly monotone mapping. Then, it follows from Lemma 2 that for each $r>0$ and $x \in H$ there is $w \in C$ such that

$$
T_{r}(x)=\{w\}
$$

where $T_{r} x=\left\{z \in C: F(z, y)+\frac{1}{r}\langle y-z, z-x\rangle \geq 0, \forall y \in C\right\}=\{w\}$, so that we identify $T_{r} x$ as simply $w$.

Let $f: C \rightarrow C$ be $\rho$-contraction mapping and let $T: C \rightarrow C$ be asymptotically nonexpansive mapping. Let $\left\{\alpha_{n}\right\} \subset[0,1]$ and $\lambda_{n} \in(0,2 \alpha)$. For any $x_{1} \in C$, we find $z_{1} \in C$ such that

$$
z_{1}=T_{\lambda_{1}}\left(x_{1}-\lambda_{1} A x_{1}\right) .
$$

Then, we can compute $x_{2} \in C$ by

$$
x_{2}=\alpha_{1} f\left(x_{1}\right)+\left(1-\alpha_{1}\right) T z_{1} .
$$

Also, we can find $z_{2} \in C$ such that

$$
z_{2}=T_{\lambda_{2}}\left(x_{2}-\lambda_{2} A x_{2}\right) .
$$

After that, we can compute $x_{3} \in C$ by

$$
x_{3}=\alpha_{2} f\left(x_{2}\right)+\left(1-\alpha_{2}\right) T^{2} z_{2} .
$$

Inductively, we can generate the sequence $\left\{x_{n}\right\} \subset C$ as follows:

$$
\left\{\begin{array}{l}
x_{1} \in C \\
z_{n}=T_{\lambda_{n}}\left(x_{n}-\lambda_{n} A x_{n}\right), n=1,2,3, \ldots \\
x_{n+1}=\alpha_{n} f\left(x_{n}\right)+\left(1-\alpha_{n}\right) T^{n} z_{n}, n=1,2,3, \ldots
\end{array}\right.
$$

Now, we state and prove our convergence theorem as follows:

Theorem 2 Let $C$ be a nonempty closed convex subset of a real Hilbert space $H$ and let $F: C \times C \rightarrow \mathbb{R}$ be a bifunction satisfying (A1), (A2), (A3), and (A4). Let $f: C \rightarrow C$ be $\rho$-contraction mapping, $A: C \rightarrow H$ be an $\alpha$-inverse strongly monotone mapping, and $T: C \rightarrow C$ be asymptotically nonexpansive mapping. Assume that $T$ is asymptotically regular on $C$ such that $F(T) \cap E P(F, A) \neq \emptyset$. Let $\left\{\alpha_{n}\right\} \subset[0,1]$ and $\left\{\lambda_{n}\right\} \subset[0,2 \alpha]$ satisfy

(i) $\lim _{n \rightarrow \infty} \alpha_{n}=0, \sum_{n=1}^{\infty} \alpha_{n}=\infty$,

(ii) $0<a \leq \lambda_{n} \leq b<2 \alpha$,

(iii) $\lim _{n \rightarrow \infty}\left(\lambda_{n}-\lambda_{n+1}\right)=0$,

(iv) $\lim _{n \rightarrow \infty} \frac{k_{n}-1}{\alpha_{n}}=0$.

For $x_{1} \in C$, if $\left\{x_{n}\right\}$ is the sequence defined by the iterative scheme (5), then $\left\{x_{n}\right\}$ converges strongly to $z=P_{F(T) \cap E P(F, A)} f(z)$. 
Proof We first show that $\left\{x_{n}\right\}$ is bounded. Let $z \in F(T) \cap E P(F, A)$. Since $z=T_{\lambda_{n}}(z-$ $\left.\lambda_{n} A z\right), A$ is $\alpha$-inverse strongly monotone and $0<\lambda_{n} \leq 2 \alpha$ for all $n \in \mathbb{N}$, we have

$$
\begin{aligned}
\left\|z_{n}-z\right\|^{2} & =\left\|T_{\lambda_{n}}\left(x_{n}-\lambda_{n} A x_{n}\right)-T_{\lambda_{n}}\left(z-\lambda_{n} A z\right)\right\|^{2} \\
& \leq\left\|\left(x_{n}-\lambda_{n} A x_{n}\right)-\left(z-\lambda_{n} A z\right)\right\|^{2} \\
& =\left\|\left(x_{n}-z\right)-\lambda_{n}\left(A x_{n}-A z\right)\right\|^{2} \\
& =\left\|x_{n}-z\right\|^{2}-2 \lambda_{n}\left\langle x_{n}-z, A x_{n}-A z\right\rangle+\lambda_{n}^{2}\left\|A x_{n}-A z\right\|^{2} \\
& \leq\left\|x_{n}-z\right\|^{2}-2 \lambda_{n} \alpha\left\|A x_{n}-A z\right\|^{2}+\lambda_{n}^{2}\left\|A x_{n}-A z\right\|^{2} \\
& =\left\|x_{n}-z\right\|^{2}+\lambda_{n}\left(\lambda_{n}-2 \alpha\right)\left\|A x_{n}-A z\right\|^{2} \\
& \leq\left\|x_{n}-z\right\|^{2} .
\end{aligned}
$$

Hence, we have

$$
\left\|z_{n}-z\right\| \leq\left\|x_{n}-z\right\|
$$

Take $\epsilon \in(0,1-\rho)$. Since $\frac{k_{n}-1}{\alpha_{n}} \rightarrow 0$ as $n \rightarrow \infty$, there exists $N \in \mathbb{N}$ such that

$$
\left(k_{n}-1\right)<\epsilon \alpha_{n} \text { for all } n \geq \mathbb{N} .
$$

From (5) and (6) it follows that, for all $n>N$

$$
\begin{aligned}
\left\|x_{n+1}-z\right\| & =\left\|\alpha_{n} f\left(x_{n}\right)+\left(1-\alpha_{n}\right) T^{n} z_{n}-z\right\| \\
& =\left\|\alpha_{n} f\left(x_{n}\right)-\alpha_{n} f(z)+\alpha_{n} f(z)-\alpha_{n} z+\alpha_{n} z+\left(1-\alpha_{n}\right) T^{n} z_{n}-z\right\| \\
& =\left\|\alpha_{n}\left(f\left(x_{n}\right)-f(z)\right)+\alpha_{n}(f(z)-z)+\left(1-\alpha_{n}\right)\left(T^{n} z_{n}-z\right)\right\| \\
& \leq \alpha_{n}\left\|f\left(x_{n}\right)-f(z)\right\|+\alpha_{n}\|f(z)-z\|+\left(1-\alpha_{n}\right)\left\|T^{n} z_{n}-z\right\| \\
& \leq \alpha_{n} \rho\left\|x_{n}-z\right\|+\alpha_{n}\|f(z)-z\|+\left(1-\alpha_{n}\right)\left\|T^{n} z_{n}-z\right\| \\
& \leq \alpha_{n} \rho\left\|x_{n}-z\right\|+\alpha_{n}\|f(z)-z\|+\left(1-\alpha_{n}\right) k_{n}\left\|z_{n}-z\right\| \\
& \leq \alpha_{n} \rho\left\|x_{n}-z\right\|+\alpha_{n}\|f(z)-z\|+\left(1-\alpha_{n}\right) k_{n}\left\|x_{n}-z\right\| \\
& =\left(1-\alpha_{n}(1-\rho)\right)\left\|x_{n}-z\right\|+\alpha_{n}\|f(z)-z\|+\left(1-\alpha_{n}\right)\left(k_{n}-1\right)\left\|x_{n}-z\right\| \\
& \leq\left(1-\alpha_{n}(1-\rho)\right)\left\|x_{n}-z\right\|+\alpha_{n}\|f(z)-z\|+\alpha_{n} \epsilon\left\|x_{n}-z\right\| \\
& =\left(1-\alpha_{n}(1-\rho-\epsilon)\right)\left\|x_{n}-z\right\|+\alpha_{n}\|f(z)-z\| \\
& \leq \max \left\{\left\|x_{n}-z\right\|, \frac{1}{1-\rho-\epsilon}\|f(z)-z\|\right\} .
\end{aligned}
$$

By induction, we see that, for all $n \geq 1$

$$
\left\|x_{n}-z\right\| \leq \max \left\{\left\|x_{1}-z\right\|, \frac{1}{1-\rho-\epsilon}\|f(z)-z\|\right\} .
$$

So $\left\{x_{n}\right\}$ is bounded, hence $\left\{A x_{n}\right\},\left\{f\left(x_{n}\right)\right\},\left\{z_{n}\right\}$ and $\left\{T^{n} z_{n}\right\}$ are bounded.

Next, we have to prove that

$$
\lim _{n \rightarrow \infty}\left\|x_{n+1}-x_{n}\right\|=0 .
$$


Since $I-\lambda_{n} A$ is non-expansive and by Lemma 3, then we have

$$
\begin{aligned}
\left\|z_{n+1}-z_{n}\right\| & =\left\|T_{\lambda_{n+1}}\left(x_{n+1}-\lambda_{n+1} A x_{n+1}\right)-T_{\lambda_{n}}\left(x_{n}-\lambda_{n} A x_{n}\right)\right\| \\
& \leq\left\|\left(x_{n+1}-\lambda_{n+1} A x_{n+1}\right)-\left(x_{n}-\lambda_{n} A x_{n}\right)\right\| \\
& +\left|\frac{\lambda_{n+1}-\lambda_{n}}{\lambda_{n+1}}\right|\left\|T_{\lambda_{n+1}}\left(x_{n+1}-\lambda_{n+1} A x_{n+1}\right)-\left(x_{n+1}-\lambda_{n+1} A x_{n+1}\right)\right\| \\
& =\left\|\left(x_{n+1}-\lambda_{n+1} A x_{n+1}\right)-\left(x_{n}-\lambda_{n+1} A x_{n}\right)+\left(\lambda_{n}-\lambda_{n+1}\right) A x_{n}\right\| \\
& +\left|\frac{\lambda_{n+1}-\lambda_{n}}{\lambda_{n+1}}\right|\left\|T_{\lambda_{n+1}}\left(x_{n+1}-\lambda_{n+1} A x_{n+1}\right)-\left(x_{n+1}-\lambda_{n+1} A x_{n+1}\right)\right\| \\
& \leq\left\|\left(x_{n+1}-\lambda_{n+1} A x_{n+1}\right)-\left(x_{n}-\lambda_{n+1} A x_{n}\right)\right\|+\left|\lambda_{n}-\lambda_{n+1}\right|\left\|A x_{n}\right\| \\
& +\left|\frac{\lambda_{n+1}-\lambda_{n}}{\lambda_{n+1}}\right|\left\|T_{\lambda_{n+1}}\left(x_{n+1}-\lambda_{n+1} A x_{n+1}\right)-\left(x_{n+1}-\lambda_{n+1} A x_{n+1}\right)\right\| \\
& \leq\left\|x_{n+1}-x_{n}\right\|+\left|\lambda_{n}-\lambda_{n+1}\right|\left\|A x_{n}\right\| \\
& +\left|\frac{\lambda_{n+1}-\lambda_{n}}{\lambda_{n+1}}\right|\left\|T_{\lambda_{n+1}}\left(x_{n+1}-\lambda_{n+1} A x_{n+1}\right)-\left(x_{n+1}-\lambda_{n+1} A x_{n+1}\right)\right\| \\
& \leq\left\|x_{n+1}-x_{n}\right\|+\left|\lambda_{n}-\lambda_{n+1}\right|\left\|A x_{n}\right\|+\frac{\left|\lambda_{n+1}-\lambda_{n}\right|}{\lambda_{n+1}} P_{n+1}
\end{aligned}
$$

where by $P_{n+1}=\sup \left\{\left\|T_{\lambda_{n+1}}\left(x_{n+1}-\lambda_{n+1} A x_{n+1}\right)-\left(x_{n+1}-\lambda_{n+1} A x_{n+1}\right)\right\|\right\}$.

On the other hand, from $z_{n}=T_{\lambda_{n}}\left(x_{n}-\lambda_{n} A x_{n}\right)$ and $z_{n+1}=T_{\lambda_{n+1}}\left(x_{n+1}-\lambda_{n+1} A x_{n+1}\right)$, we have

$$
F\left(z_{n}, y\right)+\left\langle A x_{n}, y-z_{n}\right\rangle+\frac{1}{\lambda_{n}}\left\langle y-z_{n}, z_{n}-x_{n}\right\rangle \geq 0, \forall y \in C .
$$

and

$$
F\left(z_{n+1}, y\right)+\left\langle A x_{n+1}, y-z_{n+1}\right\rangle+\frac{1}{\lambda_{n+1}}\left\langle y-z_{n+1}, z_{n+1}-x_{n+1}\right\rangle \geq 0, \forall y \in C .
$$

Putting $y=z_{n+1}$ in (8) and $y=z_{n}$ in (9), we have

$$
F\left(z_{n}, z_{n+1}\right)+\left\langle A x_{n}, z_{n+1}-z_{n}\right\rangle+\frac{1}{\lambda_{n}}\left\langle z_{n+1}-z_{n}, z_{n}-x_{n}\right\rangle \geq 0 .
$$

and

$$
F\left(z_{n+1}, z_{n}\right)+\left\langle A x_{n+1}, z_{n}-z_{n+1}\right\rangle+\frac{1}{\lambda_{n+1}}\left\langle z_{n}-z_{n+1}, z_{n+1}-x_{n+1}\right\rangle \geq 0 .
$$

So, from (A2), we have,

$$
\left\langle A x_{n+1}-A x_{n}, z_{n}-z_{n+1}\right\rangle+\left\langle z_{n+1}-z_{n}, \frac{z_{n}-x_{n}}{\lambda_{n}}-\frac{z_{n+1}-x_{n+1}}{\lambda_{n+1}}\right\rangle \geq 0 .
$$

And hence,

$$
\begin{aligned}
0 & \leq\left\langle z_{n}-z_{n+1}, \lambda_{n}\left(A x_{n+1}-A x_{n}\right)+\frac{\lambda_{n}}{\lambda_{n+1}}\left(z_{n+1}-x_{n+1}\right)-\left(z_{n}-x_{n}\right)\right\rangle \\
& =\left\langle z_{n+1}-z_{n}, z_{n}-z_{n+1}+\left(1-\frac{\lambda_{n}}{\lambda_{n+1}}\right) z_{n+1}+\left(x_{n+1}-\lambda_{n} A x_{n+1}\right)\right\rangle \\
& +\left\langle z_{n+1}-z_{n},\left(\lambda_{n} A x_{n}-x_{n}\right)-x_{n+1}+\frac{\lambda_{n}}{\lambda_{n+1}} x_{n+1}\right\rangle \\
& =\left\langle z_{n+1}-z_{n}, z_{n}-z_{n+1}+\left(1-\frac{\lambda_{n}}{\lambda_{n+1}}\right)\left(z_{n+1}-x_{n+1}\right)\right\rangle \\
& +\left\langle z_{n+1}-z_{n},\left(x_{n+1}-\lambda_{n} A x_{n+1}\right)-\left(x_{n}-\lambda_{n} A x_{n}\right)\right\rangle .
\end{aligned}
$$


It then follows that

$$
\left\|z_{n+1}-z_{n}\right\|^{2} \leq\left\|z_{n+1}-z_{n}\right\|\left\{\left|1-\frac{\lambda_{n}}{\lambda_{n+1}}\right|\left\|z_{n+1}-x_{n+1}\right\|+\left\|x_{n+1}-x_{n}\right\|\right\}
$$

And so, we have

$$
\left\|z_{n+1}-z_{n}\right\| \leq\left|1-\frac{\lambda_{n}}{\lambda_{n+1}}\right|\left\|z_{n+1}-x_{n+1}\right\|+\left\|x_{n+1}-x_{n}\right\| .
$$

Using condition (ii), we obtain

$$
\begin{aligned}
\left\|z_{n+1}-z_{n}\right\| & \leq\left\|x_{n+1}-x_{n}\right\|+\frac{1}{\lambda_{n+1}}\left|\lambda_{n+1}-\lambda_{n}\right|\left\|z_{n+1}-x_{n+1}\right\| \\
& \leq\left\|x_{n+1}-x_{n}\right\|+\frac{1}{a}\left|\lambda_{n+1}-\lambda_{n}\right| M,
\end{aligned}
$$

where $M=\sup _{n \geq 1}\left\|z_{n}-x_{n}\right\|$. Hence, we have

$$
\left\|z_{n}-z_{n-1}\right\| \leq\left\|x_{n}-x_{n-1}\right\|+\frac{1}{a}\left|\lambda_{n}-\lambda_{n-1}\right| M .
$$

Consider

$$
\begin{aligned}
\left\|T^{n} z_{n}-T^{n-1} z_{n-1}\right\| & \leq\left\|T^{n} z_{n}-T^{n} z_{n-1}\right\|+\left\|T^{n} z_{n-1}-T^{n-1} z_{n-1}\right\| \\
& \leq k_{n}\left\|z_{n}-z_{n-1}\right\|+\left\|T^{n} z_{n-1}-T^{n-1} z_{n-1}\right\| .
\end{aligned}
$$

From (5), (14) and (15), we have that

$$
\begin{aligned}
\left\|x_{n+1}-x_{n}\right\| & =\left\|\alpha_{n} f\left(x_{n}\right)+\left(1-\alpha_{n}\right) T^{n} z_{n}-\alpha_{n-1} f\left(x_{n-1}\right)-\left(1-\alpha_{n-1}\right) T^{n-1} z_{n-1}\right\| \\
& \leq \alpha_{n} \rho\left\|x_{n}-x_{n-1}\right\|+\left|\alpha_{n}-\alpha_{n-1}\right|\left(\| f\left(x_{n-1}\|+\| T^{n-1} z_{n-1} \|\right)\right. \\
& +\left(1-\alpha_{n}\right)\left\|T^{n} z_{n}-T^{n-1} z_{n-1}\right\| \\
& \leq \alpha_{n} \rho\left\|x_{n}-x_{n-1}\right\|+\left|\alpha_{n}-\alpha_{n-1}\right| K+\left(1-\alpha_{n}\right)\left\|T^{n} z_{n}-T^{n-1} z_{n-1}\right\| \\
& \leq \alpha_{n} \rho\left\|x_{n}-x_{n-1}\right\|+\left|\alpha_{n}-\alpha_{n-1}\right| K+\left(1-\alpha_{n}\right) k_{n}\left\|z_{n}-z_{n-1}\right\| \\
& +\left(1-\alpha_{n}\right)\left\|T^{n} z_{n-1}-T^{n-1} z_{n-1}\right\| \\
& \leq \alpha_{n} \rho\left\|x_{n}-x_{n-1}\right\|+\left|\alpha_{n}-\alpha_{n-1}\right| K+\left(1-\alpha_{n}\right)\left(k_{n}-1\right)\left\|z_{n}-z_{n-1}\right\| \\
& +\left(1-\alpha_{n}\right)\left\|T^{n} z_{n-1}-T^{n-1} z_{n-1}\right\|+\left(1-\alpha_{n}\right)\left\|z_{n}-z_{n-1}\right\| \\
& \leq \alpha_{n} \rho\left\|x_{n}-x_{n-1}\right\|+\left|\alpha_{n}-\alpha_{n-1}\right| K+\left(k_{n}-1\right)\left\|x_{n}-x_{n-1}\right\| \\
& +\left(k_{n}-1\right) \frac{1}{a}\left|\lambda_{n}-\lambda_{n-1}\right| M+\left\|T^{n} z_{n-1}-T^{n-1} z_{n-1}\right\| \\
& +\left(1-\alpha_{n}\right)\left[\left\|x_{n}-x_{n-1}\right\|+\frac{1}{a}\left|\lambda_{n}-\lambda_{n-1}\right| M\right] \\
& \leq\left(1-\alpha_{n}(1-\rho-\epsilon)\right)\left\|x_{n}-x_{n-1}\right\|+\left|\alpha_{n}-\alpha_{n-1}\right| K+\frac{\epsilon \alpha_{n}}{a}\left|\lambda_{n}-\lambda_{n-1}\right| M \\
& +\left\|T^{n} z_{n-1}-T^{n-1} z_{n-1}\right\|+\frac{\left(1-\alpha_{n}\right)}{a}\left|\lambda_{n}-\lambda_{n-1}\right| M, \\
& \leq\left(1-\alpha_{n}(1-\rho-\epsilon)\right)\left\|x_{n}-x_{n-1}\right\|+\alpha_{n}(1-\rho-\epsilon) \frac{\left|\lambda_{n}-\lambda_{n-1}\right|}{a} M \\
& +\left(1+\alpha_{n}(2 \epsilon+\rho)\right) \frac{\left|\lambda_{n}-\lambda_{n-1}\right|}{a} M+\left|\alpha_{n}-\alpha_{n-1}\right| K \\
& +\left\|T^{n} z_{n-1}-T^{n-1} z_{n-1}\right\|,
\end{aligned}
$$

where $K=\sup \left\{\left\|f\left(x_{n}\right)\right\|+\left\|T^{n} z_{n}\right\|\right\}$. Put $s_{n}=\alpha_{n}(1-\rho-\epsilon), \beta_{n}=\frac{\left|\lambda_{n}-\lambda_{n-1}\right|}{a} M$ and $\gamma_{n}=\left(1+\alpha_{n}(2 \epsilon+\rho)\right) \frac{\left|\lambda_{n}-\lambda_{n-1}\right|}{a} M+\left|\alpha_{n}-\alpha_{n-1}\right| K+\left\|T^{n} z_{n-1}-T^{n-1} z_{n-1}\right\|$. Then,

$$
\left\|x_{n+1}-x_{n}\right\| \leq\left(1-s_{n}\right)\left\|x_{n}-x_{n-1}\right\|+s_{n} \beta_{n}+\gamma_{n}
$$


Using Lemma 4, we have

$$
\lim _{n \rightarrow \infty}\left\|x_{n+1}-x_{n}\right\|=0
$$

Further by (13) with the condition that $\lim _{n \rightarrow \infty}\left(\lambda_{n}-\lambda_{n+1}\right)=0$, we get

$$
\lim _{n \rightarrow \infty}\left\|z_{n+1}-z_{n}\right\|=0 .
$$

Since $x_{n}=\alpha_{n-1} f\left(x_{n-1}\right)+\left(1-\alpha_{n-1}\right) T^{n-1} z_{n-1}$, we have

$$
\begin{aligned}
\left\|x_{n}-T^{n} z_{n}\right\| & \leq\left\|x_{n}-T^{n-1} z_{n-1}\right\|+\left\|T^{n-1} z_{n-1}-T^{n} z_{n}\right\| \\
& \leq\left\|x_{n}-T^{n-1} z_{n-1}\right\|+\left\|T^{n-1} z_{n-1}-T^{n} z_{n-1}\right\|+\left\|T^{n} z_{n-1}-T^{n} z_{n}\right\| \\
& \leq \alpha_{n-1}\left\|f\left(x_{n-1}\right)-T^{n-1} z_{n-1}\right\| \\
& +\left\|T^{n-1} z_{n-1}-T^{n} z_{n-1}\right\|+k_{n}\left\|z_{n-1}-z_{n}\right\| .
\end{aligned}
$$

From (17) with $\alpha_{n} \rightarrow 0$ as $n \rightarrow \infty$ and $T$ is asymptotically regular on $C$.

It follows that

$$
\lim _{n \rightarrow \infty}\left\|T^{n} z_{n}-x_{n}\right\|=0
$$

Now, we have to prove that

$$
\lim _{n \rightarrow \infty}\left\|T x_{n}-x_{n}\right\|=0 .
$$

To show this, we first prove that

$$
\lim _{n \rightarrow \infty}\left\|z_{n}-x_{n}\right\|=0 .
$$

With the fact that A is $\alpha$-inverse strongly monotone, let us consider the following:

$$
\begin{aligned}
\left\|z_{n}-z\right\|^{2} & =\left\|T_{\lambda_{n}}\left(I-\lambda_{n} A\right) x_{n}-T_{\lambda_{n}}\left(I-\lambda_{n} A\right) z\right\|^{2} \\
& \leq\left\|\left(I-\lambda_{n} A\right) x_{n}-\left(I-\lambda_{n} A\right) z\right\|^{2} \\
& =\|\left(x_{n}-z\right)-\lambda_{n}\left(A x_{n}-A z \|^{2}\right. \\
& =\left\|x_{n}-z\right\|^{2}-2 \lambda_{n}\left\langle x_{n}-z, A x_{n}-A z\right\rangle+\lambda_{n}^{2}\left\|A x_{n}-A z\right\|^{2} \\
& \leq\left\|x_{n}-z\right\|^{2}-2 \lambda_{n} \alpha\left\|A x_{n}-A z\right\|^{2}+\lambda_{n}^{2}\left\|A x_{n}-A z\right\|^{2} \\
& =\left\|x_{n}-z\right\|^{2}+\lambda_{n}\left(\lambda_{n}-2 \alpha\right)\left\|A x_{n}-A z\right\|^{2} .
\end{aligned}
$$

From the convexity of $\|\cdot\|^{2}$, (18), and (19), we have

$$
\begin{aligned}
\left\|x_{n+1}-z\right\|^{2} & =\left\|\alpha_{n} f\left(x_{n}\right)-\alpha_{n} z+\alpha_{n} z+\left(1-\alpha_{n}\right) T^{n} z_{n}-z\right\|^{2} \\
& =\left\|\alpha_{n}\left(f\left(x_{n}\right)-z\right)+\left(1-\alpha_{n}\right)\left(T^{n} z_{n}-z\right)\right\|^{2} \\
\leq & \alpha_{n} \|\left(f\left(x_{n}\right)-z\left\|^{2}+\left(1-\alpha_{n}\right)\right\| T^{n} z_{n}-z \|^{2}\right. \\
\leq & \alpha_{n} \|\left(f\left(x_{n}\right)-z\left\|^{2}+\left(1-\alpha_{n}\right) k_{n}^{2}\right\| z_{n}-z \|^{2}\right. \\
\leq & \alpha_{n}\left\|f\left(x_{n}\right)-z\right\|^{2}+\left(1-\alpha_{n}\right) k_{n}^{2}\left[\left\|x_{n}-z\right\|^{2}\right. \\
& \left.+\lambda_{n}\left(\lambda_{n}-2 \alpha\right)\left\|A x_{n}-A z\right\|^{2}\right] \\
& =\alpha_{n}\left\|f\left(x_{n}\right)-z\right\|^{2}+\left(1-\alpha_{n}\right) k_{n}^{2}\left\|x_{n}-z\right\|^{2} \\
& +\lambda_{n}\left(\lambda_{n}-2 \alpha\right)\left(1-\alpha_{n}\right) k_{n}^{2}\left\|A x_{n}-A z\right\|^{2} \\
& =\alpha_{n}\left\|f\left(x_{n}\right)-z\right\|^{2}+\left(1-\alpha_{n}\right)\left(k_{n}^{2}-1\right)\left\|x_{n}-z\right\|^{2} \\
& +\left(1-\alpha_{n}\right)\left\|x_{n}-z\right\|^{2}+\lambda_{n}\left(\lambda_{n}-2 \alpha\right)\left(1-\alpha_{n}\right) k_{n}^{2}\left\|A x_{n}-A z\right\|^{2} \\
\leq & \alpha_{n}\left\|f\left(x_{n}\right)-z\right\|^{2}+\left(1-\alpha_{n}\right)\left(k_{n}^{2}-1\right)\left\|x_{n}-z\right\|^{2}+\left\|x_{n}-z\right\|^{2} \\
& +\lambda_{n}\left(\lambda_{n}-2 \alpha\right)\left(1-\alpha_{n}\right) k_{n}^{2}\left\|A x_{n}-A z\right\|^{2}
\end{aligned}
$$


which implies that

$$
\begin{aligned}
\lambda_{n}\left(2 \alpha-\lambda_{n}\right)\left(1-\alpha_{n}\right) k_{n}^{2}\left\|A x_{n}-A z\right\|^{2} & \leq \alpha_{n}\left\|f\left(x_{n}\right)-z\right\|^{2}+\left(1-\alpha_{n}\right)\left(k_{n}^{2}-1\right)\left\|x_{n}-z\right\|^{2} \\
& +\left\|x_{n}-z\right\|^{2}-\left\|x_{n+1}-z\right\|^{2} .
\end{aligned}
$$

Since $\lim _{n \rightarrow \infty} \alpha_{n}=0, \lim _{n \rightarrow \infty} k_{n}=1$ and both $\left\{f\left(x_{n}\right)\right\}$ and $\left\{x_{n}\right\}$ are bounded by (16), we have

$$
\lim _{n \rightarrow \infty}\left\|A x_{n}-A z\right\|=0 .
$$

Since $\left(I-\lambda_{n} A\right)$ is non-expansive and by Lemma 2 , we have

$$
\begin{aligned}
\left\|z_{n}-z\right\|^{2}= & \left\|T_{\lambda_{n}}\left(x_{n}-\lambda_{n} A x_{n}\right)-T_{\lambda_{n}}\left(z-\lambda_{n} A z\right)\right\|^{2} \\
\leq & \left\langle z_{n}-z,\left(x_{n}-\lambda_{n} A x_{n}\right)-\left(z-\lambda_{n} A z\right)\right\rangle \\
= & \frac{1}{2}\left(\left\|\left(x_{n}-\lambda_{n} A x_{n}\right)-\left(z-\lambda_{n} A z\right)\right\|^{2}+\left\|z_{n}-z\right\|^{2}\right) \\
& -\frac{1}{2}\left(\left\|\left(x_{n}-\lambda_{n} A x_{n}\right)-\left(z-\lambda_{n} A z\right)-\left(z_{n}-z\right)\right\|^{2}\right) \\
= & \frac{1}{2}\left(\left\|\left(I-\lambda_{n} A\right) x_{n}-\left(I-\lambda_{n} A\right) z\right\|^{2}+\left\|z_{n}-z\right\|^{2}\right) \\
& -\frac{1}{2}\left(\left\|\left(x_{n}-z_{n}\right)-\lambda_{n}\left(A x_{n}-A z\right)\right\|^{2}\right) \\
\leq & \frac{1}{2}\left(\left\|x_{n}-z\right\|^{2}+\left\|z_{n}-z\right\|^{2}-\left\|\left(x_{n}-z_{n}\right)-\lambda_{n}\left(A x_{n}-A z\right)\right\|^{2}\right) \\
\leq & \frac{1}{2}\left(\left\|x_{n}-z\right\|^{2}+\left\|z_{n}-z\right\|^{2}-\left\|x_{n}-z_{n}\right\|^{2}+2 \lambda_{n}\left\langle x_{n}-z_{n}, A x_{n}-A z\right\rangle\right) \\
- & \frac{1}{2} \lambda_{n}^{2}\left\|A x_{n}-A z\right\|^{2}
\end{aligned}
$$

which implies that

$$
\left\|z_{n}-z\right\|^{2} \leq\left\|x_{n}-z\right\|^{2}-\left\|x_{n}-z_{n}\right\|^{2}+2 \lambda_{n}\left\langle x_{n}-z_{n}, A x_{n}-A z\right\rangle-\lambda_{n}^{2}\left\|A x_{n}-A z\right\|^{2} .
$$

From (20) and (24), we have

$$
\begin{aligned}
\left\|x_{n+1}-z\right\|^{2} & \leq \alpha_{n}\left\|f\left(x_{n}\right)-z\right\|^{2}+\left(1-\alpha_{n}\right) k_{n}^{2}\left(\left\|x_{n}-z\right\|^{2}-\left\|x_{n}-z_{n}\right\|^{2}\right) \\
& +2 \lambda_{n}(1-\alpha) k_{n}^{2}\left(\left\langle x_{n}-z_{n}, A x_{n}-A z\right\rangle-\lambda_{n}^{2}\left\|A x_{n}-A z\right\|^{2}\right) \\
& \leq \alpha_{n}\left\|f\left(x_{n}\right)-z\right\|^{2}+\left(1-\alpha_{n}\right) k_{n}^{2}\left\|x_{n}-z\right\|^{2}-\left(1-\alpha_{n}\right) k_{n}^{2}\left\|x_{n}-z_{n}\right\|^{2} \\
& +2\left(1-\alpha_{n}\right) k_{n}^{2} \lambda_{n}\left\|x_{n}-z_{n}\right\|\left\|A x_{n}-A z\right\|-\left(1-\alpha_{n}\right) k_{n}^{2} \lambda_{n}^{2}\left\|A x_{n}-A z\right\|^{2} \\
& \leq \alpha_{n}\left\|f\left(x_{n}\right)-z\right\|^{2}+\left(1-\alpha_{n}\right) k_{n}^{2}\left\|x_{n}-z\right\|^{2}-\left(1-\alpha_{n}\right) k_{n}^{2}\left\|x_{n}-z_{n}\right\|^{2} \\
& +2\left(1-\alpha_{n}\right) k_{n}^{2} \lambda_{n}\left\|x_{n}-z_{n}\right\|\left\|A x_{n}-A z\right\| \\
& =\alpha_{n}\left\|f\left(x_{n}\right)-z\right\|^{2}+\left(1-\alpha_{n}\right)\left(k_{n}^{2}-1\right)\left\|x_{n}-z\right\|^{2}+\left(1-\alpha_{n}\right)\left\|x_{n}-z\right\|^{2} \\
& \left.-\left(1-\alpha_{n}\right) k_{n}^{2}\left\|x_{n}-z_{n}\right\|^{2}+2\left(1-\alpha_{n}\right) k_{n}^{2} \lambda_{n}\left\|x_{n}-z_{n}\right\|\left\|A x_{n}-A z\right\|\right) \\
& \leq \alpha_{n}\left\|f\left(x_{n}\right)-z\right\|^{2}+\left(1-\alpha_{n}\right)\left(k_{n}^{2}-1\right)\left\|x_{n}-z\right\|^{2}+\left\|x_{n}-z\right\|^{2} \\
& -\left(1-\alpha_{n}\right) k_{n}^{2}\left\|x_{n}-z_{n}\right\|^{2}+2\left(1-\alpha_{n}\right) k_{n}^{2} \lambda_{n}\left\|x_{n}-z_{n}\right\|\left\|A x_{n}-A z\right\| .
\end{aligned}
$$

Hence,

$$
\begin{aligned}
\left(1-\alpha_{n}\right) k_{n}^{2}\left\|x_{n}-z_{n}\right\|^{2} & \leq \alpha_{n}\left\|f\left(x_{n}\right)-z\right\|^{2}+\left(1-\alpha_{n}\right)\left(k_{n}^{2}-1\right)\left\|x_{n}-z\right\|^{2}+\left\|x_{n}-z\right\|^{2} \\
& -\left\|x_{n+1}-z\right\|^{2}+2\left(1-\alpha_{n}\right) k_{n}^{2} \lambda_{n}\left\|x_{n}-z_{n}\right\|\left\|A x_{n}-A z\right\| .
\end{aligned}
$$


Since $\alpha_{n} \rightarrow 0, k_{n} \rightarrow 1$ as $n \rightarrow \infty$ and $\left\{x_{n}\right\},\left\{z_{n}\right\}$ are bounded with $\lim _{n \rightarrow \infty}\left\|x_{n}-z\right\|^{2}-\left\|x_{n+1}-z\right\|^{2}=0$, we have

$$
\lim _{n \rightarrow \infty}\left\|x_{n}-z_{n}\right\|=0 .
$$

Combining (16) and (25) we have, $\left\|z_{n}-x_{n+1}\right\| \leq\left\|z_{n}-x_{n}\right\|+\left\|x_{n}-x_{n+1}\right\|$ which implies that

$$
\lim _{n \rightarrow \infty}\left\|z_{n}-x_{n+1}\right\|=0 .
$$

Since $\left\|T^{n} z_{n}-z_{n}\right\| \leq\left\|T^{n} z_{n}-x_{n}\right\|+\left\|x_{n}-z_{n}\right\|$ and from (18) and (25)

$$
\lim _{n \rightarrow \infty}\left\|T^{n} z_{n}-z_{n}\right\|=0 .
$$

Let $k=\sup _{n \in \mathbb{N}} k_{n}<\infty$. Consequently, by (17) and (27)

$$
\begin{aligned}
\left\|T z_{n}-z_{n}\right\| & \leq\left\|T z_{n}-T^{n+1} z_{n}\right\|+\left\|T^{n+1} z_{n}-T^{n+1} z_{n+1}\right\| \\
& +\left\|T^{n+1} z_{n+1}-z_{n+1}\right\|+\left\|z_{n+1}-z_{n}\right\| \\
& \leq k_{1}\left\|z_{n}-T^{n} z_{n}\right\|+k_{n+1}\left\|z_{n}-z_{n+1}\right\| \\
& +\left\|T^{n+1} z_{n+1}-z_{n+1}\right\|+\left\|z_{n+1}-z_{n}\right\| \\
& =k_{1}\left\|z_{n}-T^{n} z_{n}\right\|+\left(k_{n+1}+1\right)\left\|z_{n}-z_{n+1}\right\|+\left\|T^{n+1} z_{n+1}-z_{n+1}\right\| \\
& \leq k\left\|z_{n}-T^{n} z_{n}\right\|+(k+1)\left\|z_{n}-z_{n+1}\right\|+\left\|T^{n+1} z_{n+1}-z_{n+1}\right\|
\end{aligned}
$$

This implies that

$$
\lim _{n \rightarrow \infty}\left\|T z_{n}-z_{n}\right\|=0 .
$$

Further, we have the following result:

$$
\begin{aligned}
\left\|T x_{n}-x_{n}\right\| & \leq\left\|T x_{n}-T z_{n}\right\|+\left\|T z_{n}-z_{n}\right\|+\left\|z_{n}-x_{n}\right\| \\
& \leq k_{1}\left\|x_{n}-z_{n}\right\|+\left\|T z_{n}-z_{n}\right\|+\left\|z_{n}-x_{n}\right\| \\
& \leq\left(k_{1}+1\right)\left\|x_{n}-z_{n}\right\|+\left\|T z_{n}-z_{n}\right\|
\end{aligned}
$$

which implies that

$$
\lim _{n \rightarrow \infty}\left\|T x_{n}-x_{n}\right\|=0 .
$$

Since $P_{F(T) \cap E P(F, A)} f: C \rightarrow C$ is a $\rho$-contraction mapping, therefore, by Banach contraction principle, there exists a unique $z_{0} \in F(T) \cap E P(F, A)$ such that $z_{0}=$ $P_{F(T) \cap E P(F, A)} f\left(z_{0}\right)$. We shall show that

$$
\lim _{n \rightarrow \infty}\left\langle f\left(z_{0}\right)-z_{0}, x_{n}-z_{0}\right\rangle \leq 0 .
$$

Since $\left\{x_{n}\right\}$ is bounded sequence, we can choose a subsequence $\left\{x_{n_{i}}\right\}$ of $\left\{x_{n}\right\}$ such that

$$
\limsup _{n \rightarrow \infty}\left\langle f\left(z_{0}\right)-z_{0}, x_{n}-z_{0}\right\rangle=\lim _{i \rightarrow \infty}\left\langle f\left(z_{0}\right)-z_{0}, x_{n_{i}}-z_{0}\right\rangle .
$$

Without loss of generality, we may assume that $x_{n_{i}} \rightarrow \omega$. Since $C$ is closed and convex, $C$ is weakly closed. So, we have $\omega \in C$. Now, we will show that $\omega \in F(T)$. In fact, since $x_{n_{i}} \rightarrow \omega$ and $x_{n}-T x_{n} \rightarrow 0$ by Lemma 5 , we find that $\omega \in F(T)$.

Next, we show that $\omega \in E P(F, A)$. From (25), we have $z_{n_{i}} \rightarrow \omega$.

Since $z_{n}=T_{\lambda_{n}}\left(x_{n}-\lambda_{n} A x_{n}\right)$, that is

$$
F\left(z_{n}, y\right)+\left\langle A x_{n}, y-z_{n}\right\rangle+\frac{1}{\lambda_{n}}\left\langle y-z_{n}, z_{n}-x_{n}\right\rangle \geq 0, \forall y \in C \text {. From (A2), we have }
$$


$\left\langle A x_{n}, y-z_{n}\right\rangle+\frac{1}{\lambda_{n}}\left\langle y-z_{n}, z_{n}-x_{n}\right\rangle \geq F\left(y, z_{n}\right), \forall y \in C$.

Replacing $n$ with $n_{i}$ in the above inequality, we have,

$$
\left\langle A x_{n_{i}}, y-z_{n_{i}}\right\rangle+\frac{1}{\lambda_{n_{i}}}\left\langle y-z_{n_{i}}, z_{n_{i}}-x_{n_{i}}\right\rangle \geq F\left(y, z_{n_{i}}\right) .
$$

Put $z_{t}=t y+(1-t) \omega$ for all $t \in(0,1]$ and $y \in C$. Then, we have $z_{t} \in C$. So, from (31) we have

$$
\begin{aligned}
\left\langle z_{t}-z_{n_{i}}, A z_{t}\right\rangle & \geq\left\langle z_{t}-z_{n_{i}}, A z_{t}\right\rangle-\left\langle z_{t}-z_{n_{i}}, A x_{n_{i}}\right\rangle-\left\langle z_{t}-z_{n_{i}}, \frac{z_{n_{i}}-x_{n_{i}}}{\lambda_{n_{i}}}\right\rangle+F\left(z_{t}, z_{n_{i}}\right) \\
& =\left\langle z_{t}-z_{n_{i}}, A z_{t}-A z_{n_{i}}\right\rangle+\left\langle z_{t}-z_{n_{i}}, A z_{n_{i}}-A x_{n_{i}}\right\rangle \\
& -\left\langle z_{t}-z_{n_{i}}, \frac{z_{n_{i}}-x_{n_{i}}}{\lambda_{n_{i}}}\right\rangle+F\left(z_{t}, z_{n_{i}}\right) .
\end{aligned}
$$

Since $\lim _{n \rightarrow \infty}\left\|z_{n_{i}}-x_{n_{i}}\right\|=0$, we have $\lim _{n \rightarrow \infty}\left\|A z_{n_{i}}-A x_{n_{i}}\right\|=0$.

Further from monotonicity of $A$, we have $\left\langle z_{t}-z_{n_{i}}, A z_{t}-A z_{n_{i}}\right\rangle \geq 0$. It follows from (A4) that

$$
F\left(z_{t}, \omega\right) \leq \lim _{n \rightarrow \infty} F\left(z_{t}, z_{n_{i}}\right) \leq \lim _{n \rightarrow \infty}\left\langle A z_{t}, z_{t}-z_{n_{i}}\right\rangle=\left\langle A z_{t}, z_{t}-\omega\right\rangle
$$

From $(A 1),(A 4)$, and (32), we have

$$
\begin{aligned}
0=F\left(z_{t}, z_{t}\right) & \leq t F\left(z_{t}, y\right)+(1-t) F\left(z_{t}, \omega\right) \\
& \leq t F\left(z_{t}, y\right)+(1-t)\left\langle z_{t}-\omega, A z_{t}\right\rangle .
\end{aligned}
$$

But $z_{t}-\omega=t y+(1-t) \omega-\omega=t(y-\omega)$. So, we have the following $0=F\left(z_{t}, z_{t}\right) \leq$ $t F\left(z_{t}, y\right)+(1-t) t\left\langle y-\omega, A z_{t}\right\rangle$ and hence $0 \leq F\left(z_{t}, y\right)+(1-t)\left\langle y-\omega, A z_{t}\right\rangle$. Letting $t \rightarrow 0$, we have for each $y \in C$,

$$
\begin{aligned}
& 0 \leq F(\omega, y)+\langle y-\omega, A \omega\rangle . \\
& \Longrightarrow \omega \in E P(F, A) .
\end{aligned}
$$

Since $\omega \in F(T) \cap E P(F, A)$, from (30) and the property of metric projection, we have

$$
\begin{aligned}
\limsup _{n \rightarrow \infty}\left\langle f\left(z_{0}\right)-z_{0}, x_{n}-z_{0}\right\rangle & =\lim _{i \rightarrow \infty}\left\langle f\left(z_{0}\right)-z_{0}, x_{n_{i}}-z_{0}\right\rangle \\
& =\left\langle f\left(z_{0}\right)-z_{0}, \omega-z_{0}\right\rangle \leq 0 .
\end{aligned}
$$

Finally, we prove that $\lim _{n \rightarrow \infty}\left\|x_{n}-z_{0}\right\|=0$. 
From (5) and Lemma 6, we obtain

$$
\begin{aligned}
\left\|x_{n+1}-z_{0}\right\|^{2} & =\left\|\alpha_{n}\left(f\left(x_{n}\right)-z_{0}\right)+\left(1-\alpha_{n}\right)\left(T^{n} z_{n}-z_{0}\right)\right\|^{2} \\
& \leq\left(1-\alpha_{n}\right)^{2}\left\|T^{n} z_{n}-z_{0}\right\|^{2}+2\left\langle\alpha_{n}\left(f\left(x_{n}\right)-z_{0}\right), x_{n+1}-z_{0}\right\rangle \\
& =\left(1-\alpha_{n}\right)^{2}\left\|T^{n} z_{n}-z_{0}\right\|^{2}+2 \alpha_{n}\left\langle f\left(x_{n}\right)-z_{0}, x_{n+1}-z_{0}\right\rangle \\
& \leq\left[\left(1-\alpha_{n}\right) k_{n}\right]^{2}\left\|z_{n}-z_{0}\right\|^{2}+2 \alpha_{n}\left\langle f\left(x_{n}\right)-z_{0}, x_{n+1}-z_{0}\right\rangle \\
& =\left[\left(1-\alpha_{n}\right) k_{n}\right]^{2}\left\|z_{n}-z_{0}\right\|^{2}+2 \alpha_{n}\left\langle f\left(x_{n}\right)-f\left(z_{0}\right), x_{n+1}-z_{0}\right\rangle \\
& +2 \alpha_{n}\left\langle f\left(z_{0}\right)-z_{0}, x_{n+1}-z_{0}\right\rangle \\
& \leq\left[\left(1-\alpha_{n}\right) k_{n}\right]^{2}\left\|x_{n}-z_{0}\right\|^{2}+2 \alpha_{n}\left\langle f\left(x_{n}\right)-f\left(z_{0}\right), x_{n+1}-z_{0}\right\rangle \\
& +2 \alpha_{n}\left\langle f\left(z_{0}\right)-z_{0}, x_{n+1}-z_{0}\right\rangle \\
& \leq\left[\left(1-\alpha_{n}\right) k_{n}\right]^{2}\left\|x_{n}-z_{0}\right\|^{2}+2 \alpha_{n} \rho\left\|x_{n}-z_{0}\right\|\left\|x_{n+1}-z_{0}\right\| \\
& +2 \alpha_{n}\left\langle f\left(z_{0}\right)-z_{0}, x_{n+1}-z_{0}\right\rangle \\
& \leq\left[\left(1-\alpha_{n}\right) k_{n}\right]^{2}\left\|x_{n}-z_{0}\right\|^{2}+\alpha_{n} \rho\left(\left\|x_{n}-z_{0}\right\|^{2}+\left\|x_{n+1}-z_{0}\right\|^{2}\right) \\
& +2 \alpha_{n}\left\langle f\left(z_{0}\right)-z_{0}, x_{n+1}-z_{0}\right\rangle \\
& =\left[\left(1-\alpha_{n}\right)\left(k_{n}-1\right)+\left(1-\alpha_{n}\right)\right]^{2}\left\|x_{n}-z_{0}\right\|^{2}+\alpha_{n} \rho\left\|x_{n}-z_{0}\right\|^{2} \\
& +\alpha_{n} \rho\left\|x_{n+1}-z_{0}\right\|^{2}+2 \alpha_{n}\left\langle f\left(z_{0}\right)-z_{0}, x_{n+1}-z_{0}\right\rangle \\
& =\left[1-(2-\rho) \alpha_{n}+\alpha_{n}^{2}+\left(1-\alpha_{n}\right)^{2}\left(1-k_{n}\right)^{2}+\alpha_{n} \rho\left\|x_{n+1}-z_{0}\right\|^{2}\right. \\
& \left.+2\left(1-\alpha_{n}\right)^{2}\left(k_{n}-1\right)\right]\left\|x_{n}-z_{0}\right\|^{2}+2 \alpha_{n}\left\langle f\left(z_{0}\right)-z_{0}, x_{n+1}-z_{0}\right\rangle \\
\leq & {\left[1-(2-\rho) \alpha_{n}+\alpha_{n}^{2}+\left(1-k_{n}\right)^{2}+2\left(k_{n}-1\right)\right]\left\|x_{n}-z_{0}\right\|^{2} } \\
& +\alpha_{n} \rho\left\|x_{n+1}-z_{0}\right\|^{2}+2 \alpha_{n}\left\langle f\left(z_{0}\right)-z_{0}, x_{n+1}-z_{0}\right\rangle . \\
&
\end{aligned}
$$

Let $P_{n}=\sup _{n \in \mathbb{N}}\left\|x_{n}-z_{0}\right\|^{2}$, so now we have

$$
\begin{aligned}
\left\|x_{n+1}-z_{0}\right\|^{2} & \leq \frac{1-(2-\rho) \alpha_{n}}{1-\rho \alpha_{n}}\left\|x_{n}-z_{0}\right\|^{2}+\frac{\alpha_{n}^{2}+\left(k_{n}-1\right)^{2}+2\left(k_{n}-1\right)}{1-\rho \alpha_{n}} P_{n} \\
& +\frac{2 \alpha_{n}}{1-\rho \alpha_{n}}\left\langle f\left(z_{0}\right)-z_{0}, x_{n+1}-z_{0}\right\rangle \\
& =\left[1-\frac{(2-\rho) \alpha_{n}}{1-\rho \alpha_{n}}\right]\left\|x_{n}-z_{0}\right\|^{2}+\frac{\alpha_{n}^{2}+\left(k_{n}-1\right)^{2}+2\left(k_{n}-1\right)}{1-\rho \alpha_{n}} P_{n} \\
& +\frac{2 \alpha_{n}}{1-\rho \alpha_{n}}\left\langle f\left(z_{0}\right)-z_{0}, x_{n+1}-z_{0}\right\rangle \\
& =\left(1-a_{n}\right)\left\|x_{n}-z_{0}\right\|^{2}+a_{n} \beta_{n}
\end{aligned}
$$

where $\beta_{n}=\frac{\alpha_{n}^{2}+\left(k_{n}-1\right)^{2}+2\left(k_{n}-1\right)}{2(1-\rho) \alpha_{n}} P_{n}+\frac{1}{1-\rho}\left\langle f\left(z_{0}\right)-z_{0}, x_{n+1}-z_{0}\right\rangle$ and $a_{n}=\frac{2(1-\rho) \alpha_{n}}{1-\rho \alpha_{n}}$. Since $\lim _{n \rightarrow \infty} a_{n}=0, \sum_{n=0}^{\infty} a_{n}=\infty$, and $\limsup _{n \rightarrow \infty} \beta_{n} \leq 0$ by (34), then by Lemma 7 , we conclude that $\lim _{n \rightarrow \infty}\left\|x_{n}-z_{0}\right\|=0$.

\section{Applications}

Using our main theorem (Theorem 2), we obtain strong convergence theorems in Hilbert space.

Theorem 3 Let $C$ be a nonempty closed convex and bounded subset of $H$. Let $F: C \times C \rightarrow$ $\mathbb{R}$ be a bifunction satisfying (A1), (A2), (A3), and (A4). Let $f: C \rightarrow C$ be $\rho$-contraction mapping, and let $T: C \rightarrow C$ be asymptotically nonexpansive mapping. Assume that $T$ 
is asymptotically regular on $C$ such that $F(T) \cap E P(F) \neq \emptyset$. Let $\left\{\alpha_{n}\right\} \subset[0,1]$ and $\left\{\lambda_{n}\right\} \subset$ $[0,2 \alpha]$ satisfy

(i) $\lim _{n \rightarrow \infty} \alpha_{n}=0, \sum_{n=1}^{\infty} \alpha_{n}=\infty$,

(ii) $0<a \leq \lambda_{n} \leq b<2 \alpha$,

(iii) $\lim _{n \rightarrow \infty}\left(\lambda_{n}-\lambda_{n+1}\right)=0$,

(iv) $\lim _{n \rightarrow \infty} \frac{k_{n}-1}{\alpha_{n}}=0$.

For $x_{1} \in C$, if $\left\{x_{n}\right\}$ is the sequence defined by the iterative scheme (5), then $\left\{x_{n}\right\}$ converges strongly to $z=P_{F(T) \cap E P(F)} f(z)$.

Proof In theorem (2), put $A \equiv 0$. We obtain that $F\left(z_{n}, y\right)+\frac{1}{\lambda_{n}}\left\langle y-z_{n}, z_{n}-x_{n}\right\rangle \geq 0, \forall y \in C$. Then, for all $\alpha \in(0, \infty)$, we have $\langle x-y, A x-A y\rangle \geq \alpha\|A x-A y\|^{2}=0, \forall x, y \in C$. Thus, we obtain the desired result by Theorem 2 .

Theorem 4 Let $C$ be a nonempty closed convex and bounded subset of a real Hilbert space H. Let $f: C \rightarrow C$ be $\rho$-contraction mapping, $A$ be an $\alpha$-inverse strongly monotone mapping of $C$ into $H$, and $T: C \rightarrow C$ be asymptotically nonexpansive mapping. Assume that $T$ is asymptotically regular on $C$ such that $F(T) \cap V I(C, A) \neq \emptyset$. Let $\left\{\alpha_{n}\right\} \subset[0,1]$ and $\left\{\lambda_{n}\right\} \subset[0,2 \alpha]$ satisfy

(i) $\lim _{n \rightarrow \infty} \alpha_{n}=0, \sum_{n=1}^{\infty} \alpha_{n}=\infty$,

(ii) $0<a \leq \lambda_{n} \leq b<2 \alpha$,

(iii) $\lim _{n \rightarrow \infty}\left(\lambda_{n}-\lambda_{n+1}\right)=0$,

(iv) $\lim _{n \rightarrow \infty} \frac{k_{n}-1}{\alpha_{n}}=0$.

For $x_{1} \in C$, if $\left\{x_{n}\right\}$ is the sequence defined by the iterative scheme (5), then $\left\{x_{n}\right\}$ converges strongly to $z=P_{F(T) \cap V I(C, A)} f(z)$.

Proof In Theorem 2, put $F \equiv 0$. Then, we obtain that $\left\langle A x_{n}, y-z_{n}\right\rangle+\frac{1}{\lambda_{n}}\left\langle y-z_{n}, z_{n}-x_{n}\right\rangle \geq$ $0, \forall y \in C, \forall n \in \mathbb{N}$.

This implies that $\left\langle x_{n}-\lambda_{n} A x_{n}-z_{n}, z_{n}-y\right\rangle \geq 0, \forall y \in C$. So, we find that $z_{n}=P_{C}\left(x_{n}-\right.$ $\left.\lambda_{n} A x_{n}\right)$. Then, we obtain the desired result from Theorem 2 .

Browder and Patryshyn [9] introduced $k$ - strictly pseudocontractive mapping which is as follows:

A mapping $S: C \rightarrow C$ is called $k$ - strictly pseudocontractive if there exists $k \in[0,1)$ such that

$$
\|S x-S y\|^{2} \leq\|x-y\|^{2}+k\|(I-S) x-(I-S) y\|^{2}, \forall x, y \in C .
$$

Putting $A=I-S$, we know that

$$
\langle x-y, A x-A y\rangle \geq \frac{1-k}{2}\|A x-A y\|^{2}, \forall x, y \in C .
$$

By using the above definition and Theorem 2, we can obtain the following theorem.

Theorem 5 Let $C$ be a nonempty closed convex and bounded subset of a real Hilbert space $H$ and let $F: C \times C \rightarrow \mathbb{R}$ be a bi-function satisfying $(A 1-A 4)$. Let $f: C \rightarrow C$ be 
$\rho$-contraction mapping, $S$ be a k-strictly pseudo contractive mapping of $C$ into itself, and $T: C \rightarrow C$ be asymptotically non-expansive mapping. Assume that $T$ is asymptotically regular on $C$ such that $F(T) \cap E P(F, A) \neq \emptyset$, where $A=I-S$. Let $\left\{\alpha_{n}\right\} \subset[0,1]$ and $\left\{\lambda_{n}\right\} \subset[0,1-k]$ satisfy

(i) $\lim _{n \rightarrow \infty} \alpha_{n}=0, \sum_{n=1}^{\infty} \alpha_{n}=\infty$,

(ii) $0<a \leq \lambda_{n} \leq b<1-k$,

(iii) $\lim _{n \rightarrow \infty}\left(\lambda_{n}-\lambda_{n+1}\right)=0$,

(iv) $\lim _{n \rightarrow \infty} \frac{k_{n}-1}{\alpha_{n}}=0$.

For $x_{1} \in C$, if $\left\{x_{n}\right\}$ is the sequence defined by the iterative scheme (5), then $\left\{x_{n}\right\}$ converges strongly to $z=P_{F(T) \cap E P(F, A)} f(z)$.

Proof Since $A=I-S$ is $\frac{1-k}{2}$-inverse strongly monotone mapping. So, by Theorem 2, we obtain the desired result.

Remark 3 By replacing asymptotically nonexpansive mapping to nonexpansive single valued mapping, it gives an improved version of the main result due to Takahashi and Takahashi [7].

\title{
Acknowledgments
}

The authors would like to thank and acknowledge Sida-Mathematics Project at the Univeversity of Dar es Salaam for their financial support through out the preparation of this article.

\section{Authors' contributions}

All authors equally contributed in the manuscript. All authors read and approved the final manuscript.

Funding

This project is funded by the Sida-Mathematics project at the University of Dar es Salaam, Tanzania.

Availability of data and materials

Not applicable.

\section{Competing interests}

The authors declare that they have no competing interests.

\begin{abstract}
Author details
${ }^{1}$ Department of Mathematics, College of Natural and Applied Sciences, University of Dar es Salaam, Dar es Salaam, Tanzania. ${ }^{2}$ Department of Mathematics, College of Natural and Computational Science, Addis Ababa University, Adis Ababa, Ethiopia.
\end{abstract}

Received: 16 May 2019 Accepted: 23 October 2019

Published online: 07 November 2019

\section{References}

1. Goebel, K., Kirk, W. A.: A fixed point theorem for asymptotically nonexpansive mappings. Proc. Amer. Math. Soc. $\mathbf{3 5}$ $171-174(1972)$

2. Blum, E., Oettli, W.: From optimization and variational inequalities to equilibrium problems. Math. Stud. 63, 123-145 (1994)

3. Kazmi, K. R., Rizvi, S. H.: Iterative approximation of a common solution of a split equilibrium problem, a variational inequality problem and a fixed point problem. Egyptian. J. Math. Soc. 21, 44-51 (2013)

4. Meche, T. H, Sangago, M. G, Zegeye, H: Approximating a common solution of a finite family of generalized equilibrium and fixed point problems. SINET: Ethiop. Sci. J. 38(1), 17-28 (2015)

5. Moudafi, A., Théra, M.: Proximal and dynamical approaches to equilibrium problems. In: Lecture Notes in Econom. and Math. Systems, Vol. 477, pp. 187-201. Springer, (1999). https://doi.org/10.1007/978-3-642-45780-7_12

6. Zegeye, H., Meche, T. H., Sangago, M. G.: Algorithms of common solutions for a fixed point of hemicontractive-type mapping and a generalized equilibrium problem. Int. Adv. J. Math. Sci. 5(1), 20-26 (2017)

7. Takahashi, S, Takahashi, W: Strong convergennce theorem for a generalized equilibrium problem and a nonexpansive mapping in a Hilbert space. Nonlinear Anal. TMA. 69, 1025-1033 (2008)

8. Combettes, P. L., Hirstoaga, A.: Equilibrium programming in Hilbert spaces. Nonlinear J. Convex Anal. 6, 117-136 (2005) 
9. Browder, F. E., Petryshyn, W. V.: Construction of fixed points of nonlinear mappings in Hilbert space. Math J. Anal. Appl. 20(1967), 197-228

10. Cianciaruso, F., Marino, G., Muglia, L., Yao, Y.: A hybrid projection algorithm for finding solutions of mixed equilibrium problem and variational inequality problem. Fixed Point Theory Appl. 2010, 19 (2010)

11. Xu, H. K.: Iterative algorithms for nonlinear operators. Lond. J. Math. Soc. 66(2002), 240-256

12. Cho, Y. J., Zhou, H., Guo, G.: Weak and strong convergence theorems for three-step iterations with errors for asymptotically nonexpansive mappings. Comput. Math. Appl. 47(4-5), 707-717 (2004)

13. Agarwal, R. P., O'Regan, D., Sahu, D. R.: Fixed point theory for Lipschitzian-type mappings with applications. Springer, New York (2009)

14. $\mathrm{Xu}, \mathrm{H} . \mathrm{K}$ : Another control condition in an iterative method for nonexpansive mappings. Bull. Austral. Math. Soc. 65 109-113 (2002)

\section{Publisher's Note}

Springer Nature remains neutral with regard to jurisdictional claims in published maps and institutional affiliations.

\section{Submit your manuscript to a SpringerOpen ${ }^{\circ}$ journal and benefit from:}

- Convenient online submission

- Rigorous peer review

- Open access: articles freely available online

- High visibility within the field

- Retaining the copyright to your article

Submit your next manuscript at $\gg$ springeropen.com 